\title{
Visualizador de Água e Solo: Uma aplicação voltada para o gerenciamento de recursos naturais desenvolvida na plataforma Google Earth Engine
}

\author{
Matheus G. Mateus ${ }^{1}$, Fernanda P. Mota ${ }^{2}$, Marilton S. Aguiar $^{2}$, Diana F. Adamatti ${ }^{1}$ \\ ${ }^{1}$ Universidade Federal do Rio Grande (FURG) - Rio Grande, RS - Brasil \\ ${ }^{2}$ Universidade Federal de Pelotas (UFPel) - Pelotas, RS - Brasil \\ \{m2gonsalvez, nandapm2010, dianaada\}@gmail.com \\ mariltondinf.ufpel.edu.br
}

\begin{abstract}
Researchers linked water resources and services directly to economic growth and environmental sustainability. In this way, it is essential to store, analyze and understand the water's physical processes. The objective of this work is to identify and validate how the platform Google Earth Engine (GEE) can assist in the analysis of data in water resources. GEE integrates the technologies present in geographic information systems, making it interesting to develop applications aimed at natural resources. We applied the case study of this research to manage water resources of the hydrographic basin of Lagoa Mirim and Canal São Gonçalo. These data could be a powerful tool for the Hydrographic Basin Management Committee to understand better and analyze this region.
\end{abstract}

Resumo. Os recursos e serviços hídricos estão diretamente ligados ao crescimento econômico e à sustentabilidade ambiental. Devido a isso, é importante aperfeiçoarmos a coleta, a análise e a percepção dos processos físicos da água. O objetivo deste trabalho é identificar e avaliar como a plataforma Google Earth Engine pode auxiliar no contexto de análise de dados em recursos hídricos. O GEE integra as tecnologias presentes em sistemas de informação geográficas, tornando-a interessante para o desenvolvimento de aplicações voltadas para os recursos naturais. O estudo de caso desta pesquisa foi aplicado no gerenciamento de recursos hídricos da bacia hidrográfica da Lagoa Mirim e Canal São Gonçalo. Os dados disponibilizados são uma ferramenta poderosa para o Comitê de Gerenciamento das Bacias Hidrográficas, que pode entender e analisar esta região.

\section{Introdução}

Os recursos naturais são elementos necessários à vida humana. Sendo assim, o gerenciamento destes recursos é importante, pois visa melhorar a forma como terras, águas, minerais, petróleo, florestas e animais são gerenciados [Darby 2010]. Em relação ao recurso hídrico, o território brasileiro possui grandes bacias hidrográficas que apresentam inúmeros conflitos relacionados à distribuição e compartilhamento da água [Born et al. 2019]. O recurso hídrico, em uma bacia hidrográfica, é o principal elemento natural e renovável e seu gerenciamento busca possíveis soluções 
para os problemas enfrentados pelos gestores e usuários de um determinado ecossistema [Adamatti et al. 2005].

[Chacon-Pereira et al. 2018] afirmam que a escassez deste recurso não se deve somente à insuficiência física, mas também às desigualdades das relações sociais, sendo que estas são responsáveis pelo uso, alocação e conservação da água. Assim, neste contexto, alguns estudos buscam representar o processo de negociação de conflito na gestão participativa do uso da água, contribuindo para a compreensão do problema e desenvolvimento de boas práticas na educação ambiental [Jacobi and Francalanza 2005].

Antes da era do sensoriamento remoto, o levantamento no solo era o único método disponível. Esse método tradicional era caro, demorado e os resultados nem sempre eram precisos. Em contrapartida, o sensoriamento remoto fornece uma técnica espacial e temporal capaz de rastrear um registro histórico, que é útil para monitorar as informações da água em áreas que não possuem um programa de monitoramento em terra.

O sensoriamento remoto também foi aplicado para ajudar na avaliação e gerenciamento da qualidade da água, risco de inundação e identificação de danos, bem como alterações nos recursos hídricos superficiais [Dang et al. 2018]. O sensoriamento remoto fornece uma plataforma conveniente para monitorar as alterações espaço-temporais dos corpos d'água de superfície aberta, usando uma variedade de fontes de dados, como imagens do espectrômetro de imagem com resolução moderada (MODIS - do inglês, Moderate Resolution Imaging Spectroradiometer) [Nguyen et al. 2019], imagens Landsat [Nguyen et al. 2019] e imagens do Sentinel [Nguyen et al. 2019]. No entanto, estudos tradicionais em corpos d'água de superfície aberta geralmente usam menos imagens durante um período específico para uma região de grande escala, pois é difícil processar grandes quantidades de imagens de sensoriamento remoto [Wang et al. 2018].

A plataforma baseada em nuvem chamada Google Earth Engine (GEE) foi desenvolvida para facilitar a análise em larga escala de dados geoespaciais [Mutanga and Kumar 2019]. Neste momento, o GEE tem sido amplamente utilizado em aplicações em larga escala, incluindo mapeamento de terrenos urbanos e corpos d'água de superfície aberta.

O principal objetivo deste trabalho é desenvolver uma ferramenta no Google Earth Engine para análise do uso do solo e dos recursos hídricos na região da bacia no estado do Rio Grande do Sul. Neste trabalho será apresentado um estudo de caso no qual foi avaliada a disponibilidade de dados e informações disponibilizada pela GEE.

O artigo está organizado como segue. Na Seção 2 é descrita a plataforma GEE. A Seção 3 descreve a ferramenta proposta no GEE, voltado para recursos hídricos. Na Seção 4 são descritos os resultados e as discussões. Por fim, na Seção 5 são apresentadas as conclusões e os trabalhos futuros.

\section{Google Earth Engine}

O Google Earth Engine (GEE) é uma plataforma de processamento geoespacial baseada em nuvem que pode acessar e analisar com eficiência numerosas fontes com grande volume de dados (petabytes) geoespaciais (como imagens de sensoriamento remoto Landsat e MODIS, entre outros) a partir de servidores do Google em um período de tempo menor e em computadores que não tem um grande processamento [Gorelick et al. 2017]. Um 
exemplo da interface do GEE pode ser visualizado na Figura 1.

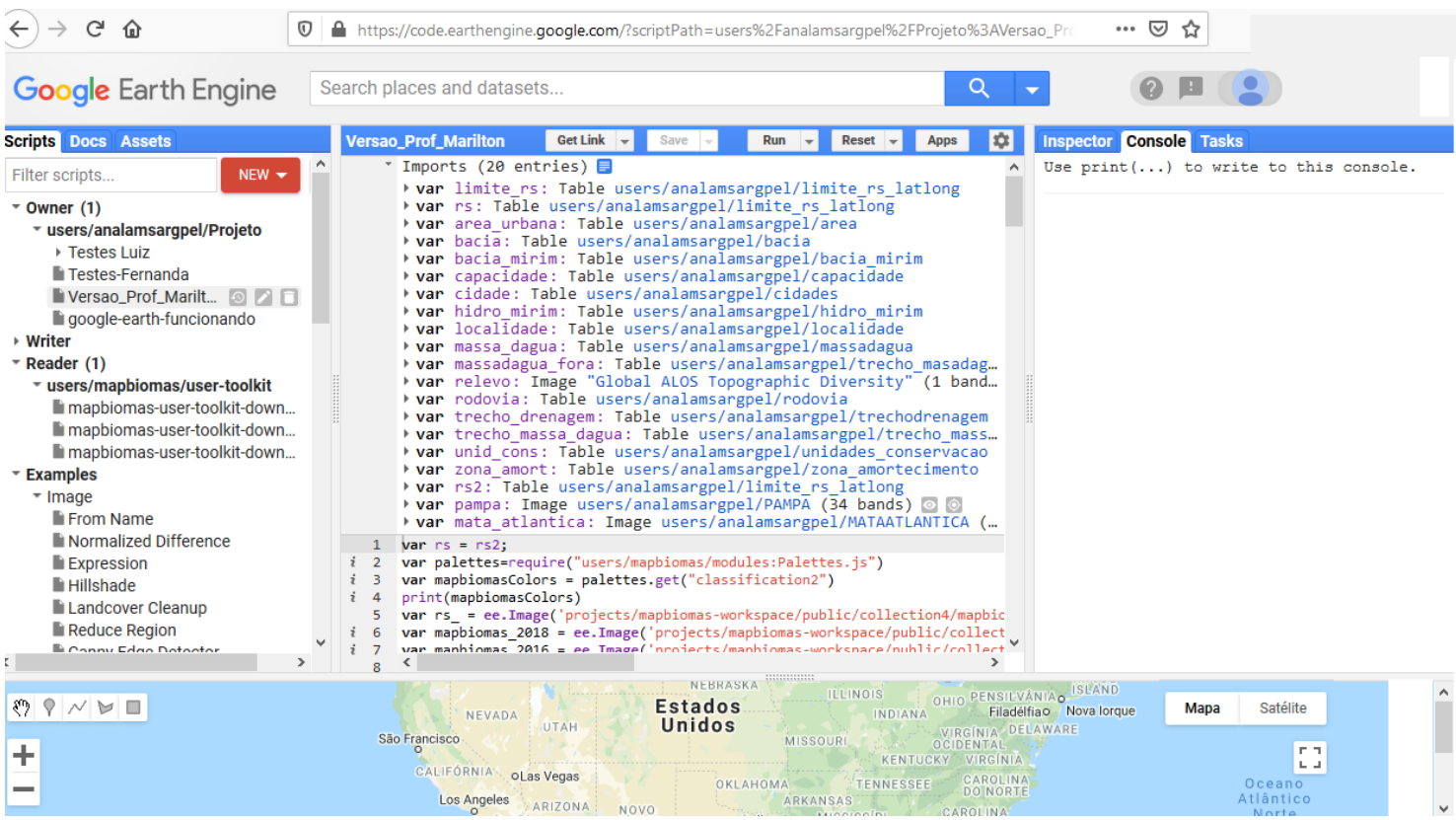

Figura 1. Plataforma GEE

O GEE utiliza um sistema de processamento paralelo para realizar o cálculo em um grande número de máquinas. Para habilitar esse processamento, o GEE utiliza as técnicas-padrão comumente usadas por linguagens funcionais, como transparência referencial e avaliação lenta, para ganhos significativos de otimização e eficiência [Gorelick et al. 2017].

Após a disponibilidade gratuita da série Landsat em 2008, o Google arquivou todos os conjuntos de dados e os vinculou ao mecanismo de computação em nuvem, para uso em código aberto. O arquivo atual de dados inclui os dados de outros satélites, bem como conjuntos de dados vetoriais baseados em sistemas de informações geográficas (GIS - Geographic Information System), modelos sociais, demográficos, climáticos, de elevação digital e camadas de dados climáticos [Gorelick et al. 2017].

Existem várias maneiras de interagir com a plataforma GEE. A linguagem de programação utilizada nesta ferramenta é o JavaScript, que facilita o desenvolvimento de aplicativos mobile, jogos e aplicações de aprendizagem de máquina. Esta plataforma é composta pelos seguintes módulos [Gorelick et al. 2017, Kumar and Mutanga 2019]: i) Code Editor é uma IDE para escrever e executar scripts; ii) Explorer é um aplicativo para explorar o catálogo de dados do GEE e executar análises simples; e, iii) bibliotecas cliente em Python e JavaScript para auxiliar os usuários no desenvolvimento de seus protótipos.

O front-end de fácil acesso fornece um ambiente conveniente para o desenvolvimento interativo de dados e algoritmos. Os usuários podem adicionar e selecionar seus próprios dados e coleções, enquanto usam os recursos de nuvem do Google para realizar todo o processamento. O GEE permite que cientistas, pesquisadores independentes e entusiastas explorem esse enorme banco de dados para detecção de mudanças, mapeamento de tendências e quantificação de recursos na superfície da Terra. A plataforma GEE não necessita de grande poder de processamento ou software muito complexo, o que permite 
a pesquisadores com poucos recursos, em países de terceiro mundo, terem a mesma capacidade de realizar análises que países desenvolvidos [Kumar and Mutanga 2019].

[Shami and Ghorbani 2019] investigaram e avaliaram a complementaridade dos dados do Landsat, Sentinel-2 e do Sentinel-1 em um ambiente no deserto de Imlili Sebkha. Os autores destacam a potencial aplicação do GEE no processamento de grandes quantidades de dados de satélite para observação de cavidades de água salgada permanentes, úmidas/secas, espaciais-temporais, livres e de longo prazo, além do monitoramento de umidade de Imlili Sebkha. Os resultados mostram que as imagens de radar não são apenas adequadas para o estudo de áreas desérticas, mas também para mapear as cavidades de água nas zonas úmidas do deserto.

[Xia et al. 2019] investigaram as mudanças climáticas nas águas superficiais da bacia do rio Huai que apresentaram impactos significativos nas plantas agrícolas, no equilíbrio ecológico e no desenvolvimento socioeconômico. Para entender as mudanças nesta região, os autores utilizaram cenas disponíveis do Landsat TM, ETM+ e do OLI no período de 1989 a 2017 e processaram os dados na plataforma GEE. Segundo os autores, compreender as variações nas áreas de corpos d'água e os fatores de controle pode apoiar a designação e implementação de práticas sustentáveis de gerenciamento de água nos usos agrícola, industrial e doméstico.

[Shami and Ghorbani 2019] analisaram o armazenamento de água superficial e subterrânea na região do Irã que tem clima quente e árido. Os autores analisaram o armazenamento de água a partir dos dados do satélite GRACE e a precipitação anual total por meio do CHIRPS no GEE no período de 2003 a 2017. Os resultados obtidos indicam uma redução nos níveis de armazenamento de água no período entre 2008 e 2017. Assim, acredita-se que o GEE é uma plataforma que pode auxiliar na análise em larga escala de dados georreferenciados relacionados aos recursos hídricos, os quais são o foco deste trabalho.

\section{Descrição das Funcionalidades da Aplicação}

O Visualizador de Água e Solo contém recursos disponíveis para análise de cobertura e uso do solo e da precipitação no estado do Rio Grande do Sul. Para a construção deste visualizador foram utilizados tanto dados nativos do GEE, que foram tratados ou obtidos diretamente de satélites, juntamente, quanto dados específicos ligados a região da Bacia Lagoa Mirim e Canal São Gonçalo, sendo a região de foco desse trabalho.

O componente Uso do solo tem como principais funções a apresentação da cobertura e uso do solo no Rio Grande do Sul ou em sub-regiões do estado e a análise de cobertura ou uso do solo limitado por região em uma determinada escala de tempo. $\mathrm{Na}$ apresentação e na análise da cobertura e uso do solo são utilizados como limites geográficos, arquivos de localidades que compõem a região da Bacia da Lagoa Mirim e Canal São Gonçalo e o limite que demarca o estado do Rio Grande do Sul, os quais são fornecidos pela Secretaria Estadual do Meio Ambiente - SEMA. Os limites geográficos utilizados neste trabalho são: i) área urbana na região da bacia; ii) áreas de conservação ambiental na bacia; iii) área total da bacia; iv) área territorial da bacia no Brasil(região que exclui todos os corpos d'água); v) cidades na região da bacia tanto no Brasil quanto no Uruguai; vi) corpos d'água da bacia(rios, lagos e lagoas ligados a bacia); vii) capacidade do uso do solo na bacia; ix) massa d'água externa à bacia (Lagoa dos Patos); x) rodovias 
que cruzam a bacia; xi) delimitação do estado do rio Grande do Sul; xii) massa d'água interna à bacia; xiii) escoamento dos Corpos d'água na bacia; xiv) perímetro ao redor das áreas de conservação ambiental.

O processamento dos dados que definem cada tipo de cobertura e uso do solo utiliza coleções fornecidas pelo Mapbiomas ${ }^{1}$, que é uma iniciativa do SEEG/OC (Sistema de Estimativas de Emissões de Gases de Efeito Estufa do Observatório do Clima) para possibilitar a análise de diferentes biomas no Brasil. O Mapbiomas foi desenvolvido por uma rede colaborativa de co-criadores formado por ONGs, universidades e empresas de tecnologia organizados por biomas e temas transversais, sendo também uma aplicação desenvolvida na plataforma GEE. Para a demonstração da cobertura e uso do solo são utilizadas as imagens referentes aos dados de integração do ano de 2018, os quais são pertencentes a coleção 4.0 para o estado do Rio Grande do Sul. As imagens utilizadas para análise pertencem a mesma coleção, porém são referentes ao dados transição do período de 1985 a 2018.

O componente Índice de chuva tem como principal função a análise da precipitação em uma determinada região, utilizando limites geográficos do estado do Rio Grande do Sul. Podendo ser feita através de dados de satélite ou das bases de dados de estações pluviométricas do estado do Rio Grande do Sul. Para indicar um índice de chuvas através de satélites, são utilizados satélites da Missão de Medição de Chuva Tropical (TRMM), uma missão conjunta entre a NASA e a Agência de Exploração Aeroespacial do Japão (JAXA), projetada para monitorar e estudar as chuvas tropicais. Tais dados pertencem a classe 3B42 que tem seus dados coletados a cada três horas no formato milímetros/hora e a classe 3B43V7 que compõe seus dados através de estimativas de dados de outros satélites incluindo a classe 3B42.

A análise de dados das bases das estações pluviométricas utiliza dados de estações dentro e fora da Bacia Lagoa Mirim e Canal São Gonçalo, os quais são fornecidos pela Agência Nacional das Águas (ANA) e pelo governo Uruguaio. As estações se encontram por todo o estado e em algumas cidades do Uruguai, seus dados são divididos em brutos e consistidos, dados que passaram por algum método de classificação mas contém lacunas nos dados coletados e brutos consistidos e preenchidos, dados com todas as lacunas preenchidas através de métodos matemáticos e análise de estações próximas.

As estações cujos dados foram coletados são: Aceguá, Arroio Grande, Canguçu, Cerro Chato, Curral Alto, Estação experimental de Piratini, Ferraria, Granja Cerrito, Granja Coronel Pedro Osório, Granja Osório, Granja Santa Maria, Herval, Madruga, Pedras Altas, Pedro Osório, Pelotas, Pinheiro Machado, Ponte Cordeiro de Farias, Santa Vitória do Palmar, Vila Freire, Bagé, Boqueirão, Estância do Espantoso, Fazenda São Jorge, Pacheca, Paraiso, Passo da Capela, Passo da Guarda CEEE, Passo do Mendonça, Passo dos Enforcados, Passo São José, Porto Tarumã, São Lourenço do Sul, Serrilhada, Torquato Severo, Torrinhas, Três Passos II, 18 de Julio, Gral. Martinez, Laguna Merin, Melo, Placido Rosas, Rio Branco, Treinta y três, Vergara. Os dados do tipo brutos e consistidos tem um intervalo particular para cada estação, já os brutos consistidos e preenchidos tem o intervalo padrão de 2001 à 2019, sendo o período definido por convenção.

\footnotetext{
${ }^{1}$ https: //mapbiomas.org/en
} 


\section{Resultados}

Nessa seção são ilustrados os recursos da aplicação, a fim de apresentar e detalhar sobre seu funcionamento. A partir dos resultados obtidos no estudo desenvolvido em [Born et al. 2020] foi definida a escolha da plataforma GEE para o desenvolvimento da ferramenta Visualizador de Água e Solo (VAS). A pesquisa desenvolvida neste trabalho é uma continuidade do que foi desenvolvido em [Mota et al. 2020]. As métricas para análise e escolha da plataforma GEE foram anteriormente detalhadas em [Born et al. 2020].

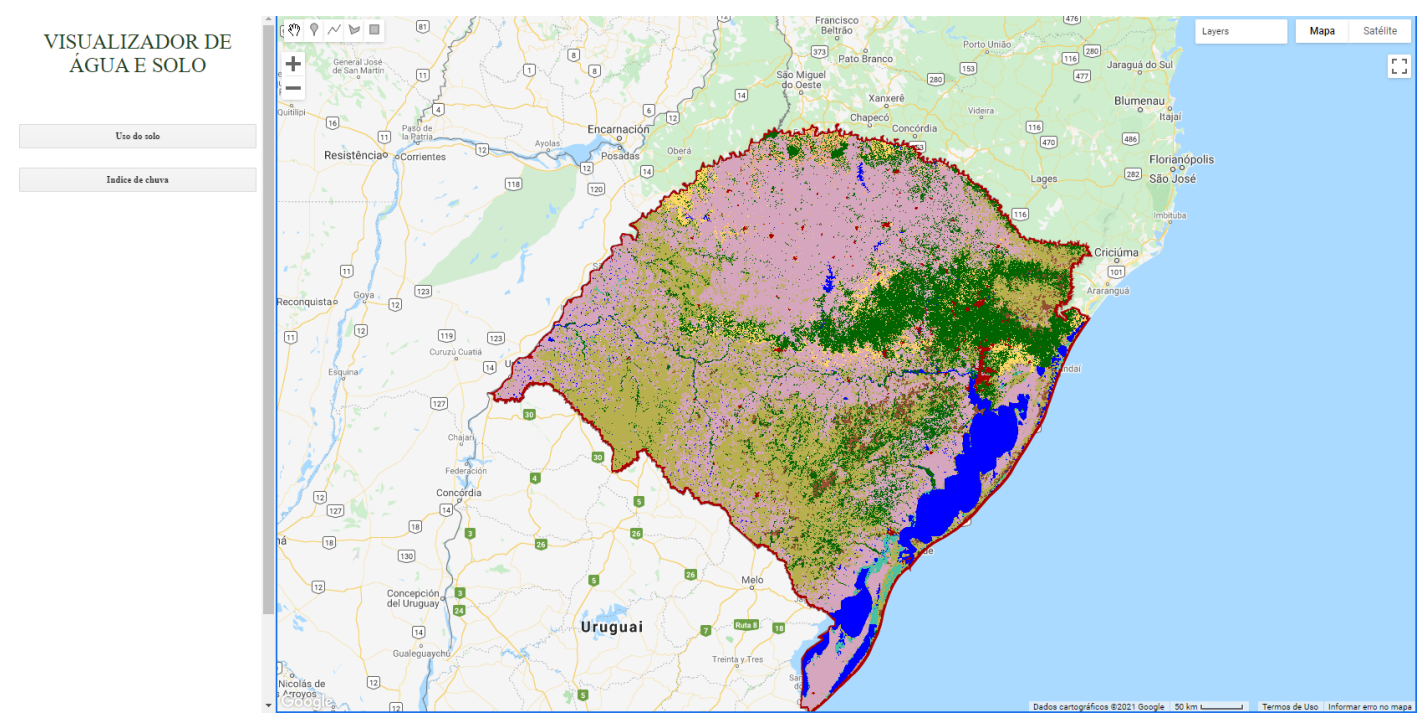

Figura 2. Tela inicial.

No menu inicial há um painel com dois botões relacionado aos componentes Uso do solo e Índice de chuva, juntamente com uma visualização da cobertura e uso do solo limitada pelo estado do Rio Grande do Sul, conforme pode ser observado na Figura 2.
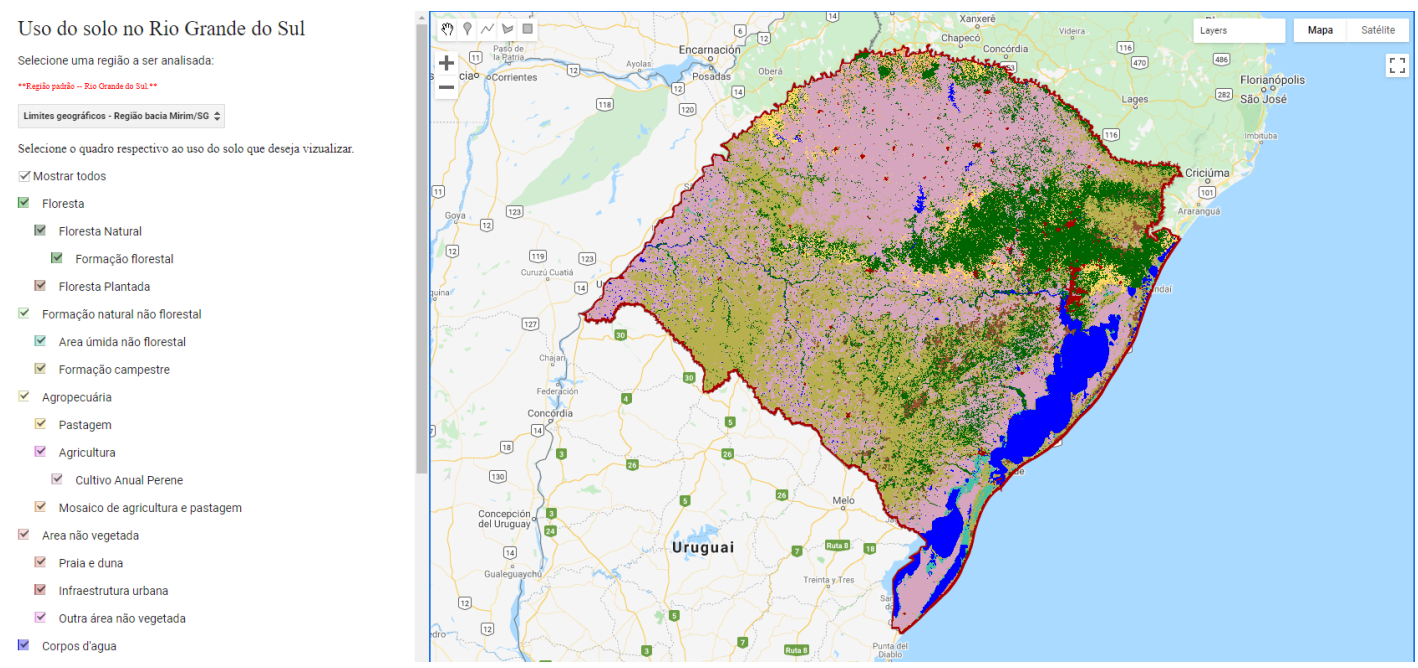

Figura 3. Uso do solo.

No componente Uso do solo (Figura 3) é possível modicar a região que limita a visualização através do menu selecionar Limites geográficos - Região bacia Mirim/SG e 
visualizar as classes de cobertura e uso do solo da forma que for mais conveniente para o usuário do sistema, através das caixas que definem quais classes estão sendo observadas no momento.

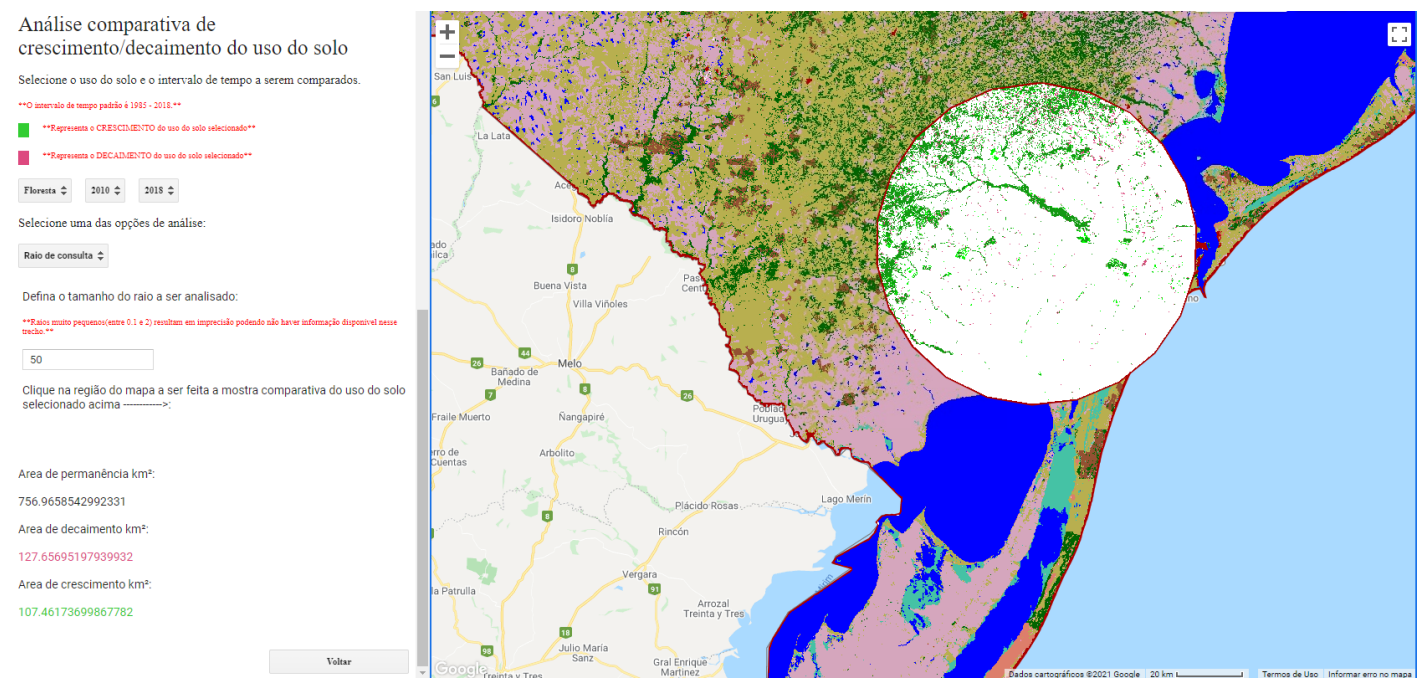

Figura 4. Uso do solo - Análise por raio de consulta.

É possível também analisar um tipo de cobertura ou uso do solo de duas maneiras: raio de consulta e área definida. Ambas necessitam que seja fornecido, além do tipo de dado, o intervalo de tempo em questão. O resultado apresentado é o decaimento, a permanência e o crescimento do tipo de cobertura ou uso do solo escolhido. O raio de consulta (Figura 4) é obtido por meio do tamanho do raio (em quilômetros) e de uma coordenada a qual centralizará esse raio, ambos fornecidos pelo usuário. O tamanho do raio é fornecido através uma caixa de texto e a coordenada fornecida através de um clique feito sobre o mapa. A área definida se refere aos limites geográficos citados ao longo do trabalho, a qual é selecionada por um outro menu selecionar Limites geográficos - Região bacia Mirim/SG e segue o mesmo padrão de mostragem do método anterior.

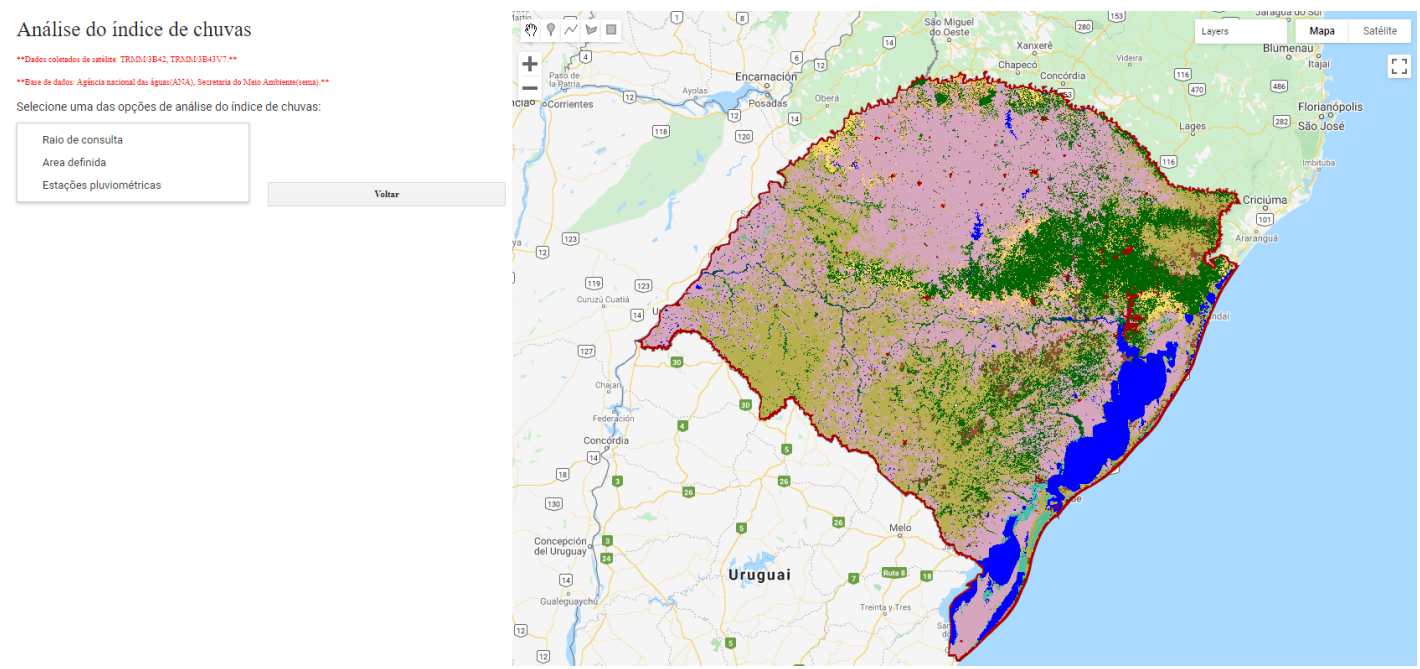

Figura 5. Índice de chuva.

No componente Índice de chuva (Figura 5) é possível fazer a análise da 
precipitação de três formas, onde duas das análises utilizam dados de satélite e a terceira os dados de precipitação das estações pluviométricas. A primeira e segunda forma consistem dos mesmos métodos para delimitar uma região utilizada na análise de cobertura e uso do solo.
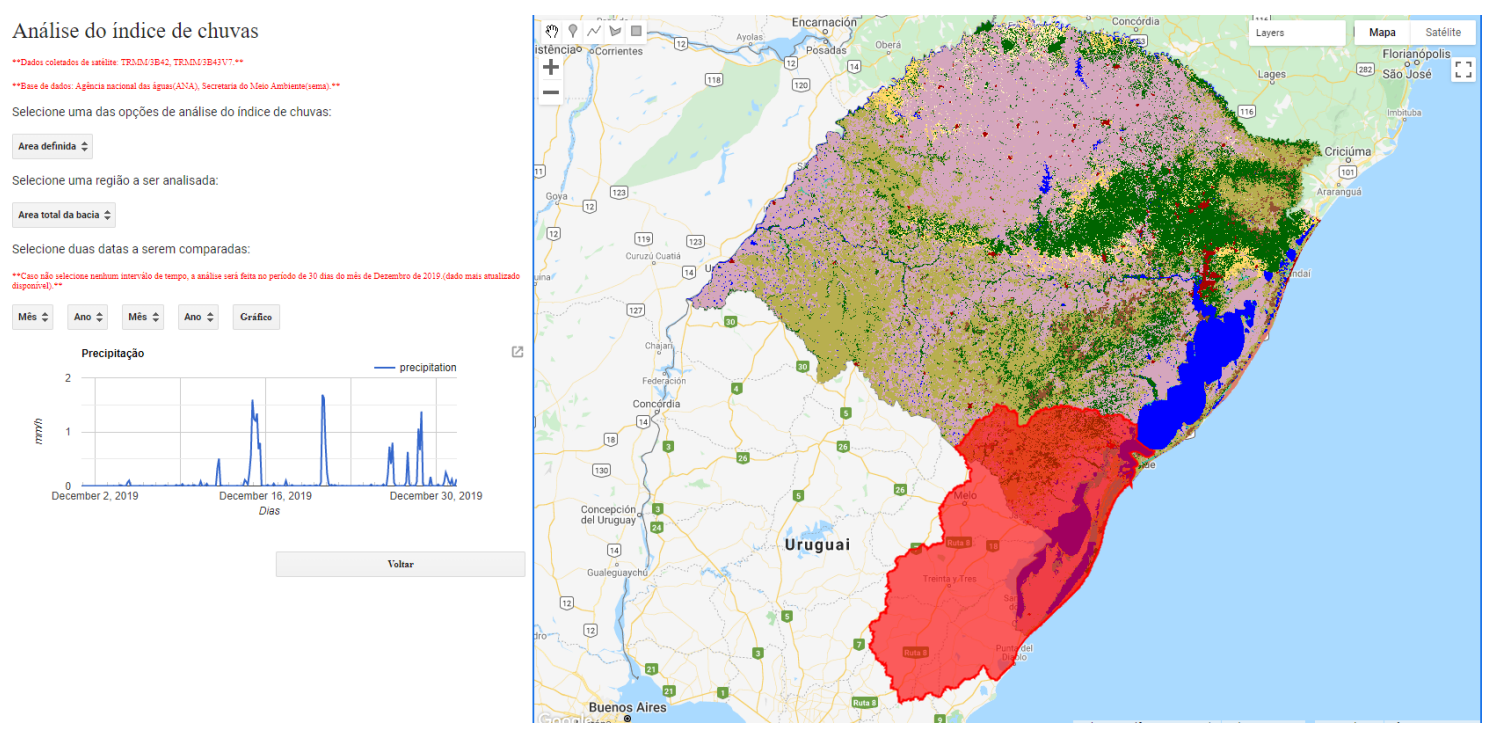

Figura 6. Índice de chuva - Análise por área definida.

As análises são feitas através de um raio de consulta ou de uma área definida (Figura 6), ambas necessitando de um período de tempo, o qual é definido por padrão o mês de dezembro de 2019, tendo como resultado o gráfico de representação da precipitação nas condições definidas.
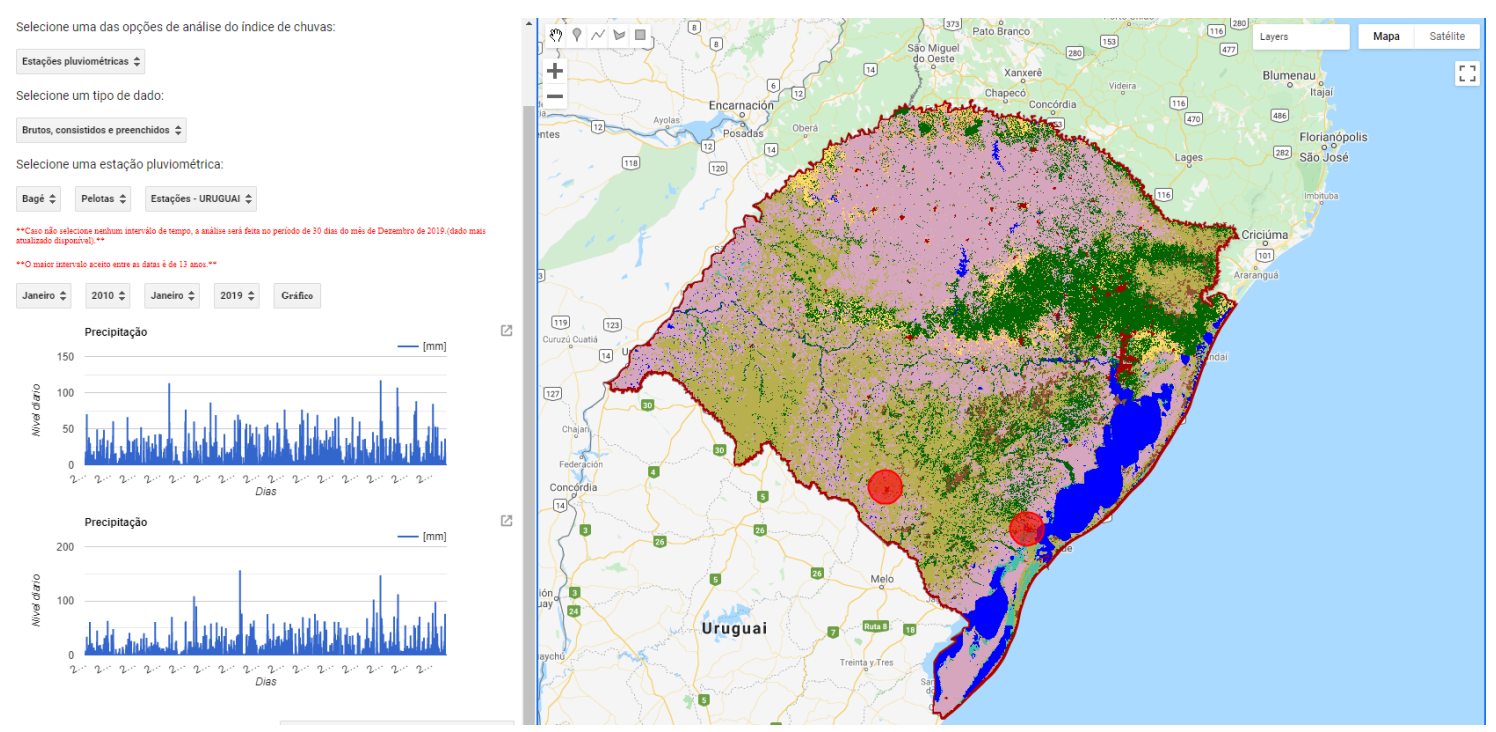

Figura 7. Índice de chuva - Análise dos dados de estações pluviométricas.

A terceira análise utiliza a localização das estações pluviométricas, representadas por uma circunferência de cinquenta quilômetros de raio, que define a região de validade de seus dados. Podendo serem analisados dados de até três estações de uma única vez, 
apresentando também um gráfico de representação da precipitação nas condições definidas para cada uma das estações, conforme pode ser observado na Figura 7.

Em todas as análises é possível para o usuário a expansão dos gráficos, para que se obtenha uma melhor análise dos dados. Também sendo disponibilizados para download nos formatos CSV, SVG e PNG. Assim, a partir deste estudo e da análise dos resultados acredita-se, que o GEE é uma plataforma que pode facilitar a análise em larga escala de dados georreferenciados relacionados aos recursos hídricos, os quais desempenham um papel crucial no crescimento econômico e na sustentabilidade ambiental.

\section{Conclusão}

A plataforma GEE se mostrou robusta para o tratamento de diferentes tipos de dados em uma mesma aplicação, possibilitando a adaptabilidade para mesclar informações de outras fontes de dados juntamente com seus dados de satélite. Não havendo nenhum tipo de interferência direta no processamento dos dados que se encontraram em tal situação. Em contraponto, ocorreram algumas restrições para alimentar a plataforma com simples tabelas de dados, dos quais continham um formato conhecido e aceito pelo GEE. Contudo ocorreram complicações na maneira como os recursos disponíveis para tratar tais dados o reconhecessem, já que simples tabelas e utilizam os mesmos recursos fornecidos para o tratamento de features, dados com estruturas mais complexas.

O processamento da plataforma é divido em cliente e servidor, consistindo na execução e processamento dos algoritmos diretamente na aplicação e fazendo requisições a processamentos feitos no servidor. Nessa aplicação foi explorado em sua grande maioria o processamento feito na parte do cliente, o qual se demonstrou completamente viável para a maior parte das análises e comparações de dados feitas em tempo real na aplicação. Porém para processar e filtrar imagens com grande quantidade de pixels, como é feito na análise de cobertura e uso do solo, utilizando apenas o processamento feito no lado do cliente, se mostrou com certas dificuldades para manter o desempenho da aplicação em determinadas densidades e extensões de pixels tratados em uma única imagem.

Como trabalhos futuros pretende-se incluir dados de estações meteorológicas pertencentes ao estado do Rio Grande do Sul, disponibilizando análises de diferentes tipos de dados como temperatura e umidade relativa do ar, temperatura do ponto de orvalho, temperatura máxima e mínima, temperatura máxima e mínima do ponto de orvalho e umidade relativa máxima e mínima do ar. Além disso, pretendemos integrar o visualizador a um sistema multiagente para controle do fluxo de água.

\section{Agradecimentos}

Os autores deste artigo agradecem a Coordenação de Aperfeiçoamento de Pessoal de Nível Superior (CAPES/Brasil) e a a Agência Nacional de Águas (ANA/Brasil) - PróRecursos Hídricos Chamada 16/2017, pelo auxílio financeiro no desenvolvimento desta pesquisa.

\section{Referências}

Adamatti, D. F., Sichman, J. S., Bommel, P., Ducrot, R., Rabak, C., and Camargo, M. (2005). Jogoman: A prototype using multi-agent-based simulation and role-playing games in water management. In Proceedings of the SMAGET-CABM-HEMA. 
Born, M., Leitzke, B. S., Farias, G., Aguiar, M., and Adamatti, D. F. (2019). Modelagem baseada em agentes para análise de recursos hídricos. In Anais do XIII WorkshopEscola de Sistemas de Agentes, seus Ambientes e apliCacoes (WESAAC 2019), pages 107-118, Florianópolis/SC. wesaac.c3.furg.br.

Born, M., Mota, F. P., Farias, G., Mateus, M. G., Leitzke, B., and Aguiar, Marilton S.and Adamatti, D. F. (2020). Estudo comparativo em gama e google earth engine:possibilidades para a área de sistemas multiagente. Remote Sensing, 1.

Chacon-Pereira, A., da Silva Batalhão, A. C., da Silva, L. P., and Neffa, E. (2018). Educação ambiental na gestão de recursos hídricos baseada no modelo de licenciamento ambiental. Desenvolvimento e Meio Ambiente, 49.

Dang, T. D., Cochrane, T. A., and Arias, M. E. (2018). Quantifying suspended sediment dynamics in mega deltas using remote sensing data: a case study of the mekong floodplains. International journal of applied earth observation and geoinformation, 68:105-115.

Darby, S. (2010). Natural resource governance: New frontiers in transparency and accountability. Transparency Accountability Initiative.

Gorelick, N., Hancher, M., Dixon, M., Ilyushchenko, S., Thau, D., and Moore, R. (2017). Google earth engine: Planetary-scale geospatial analysis for everyone. Remote sensing of Environment, 202:18-27.

Jacobi, P. R. and Francalanza, A. P. (2005). Comitês de bacias hidrográficas no brasil: desafios de fortalecimento da gestão compartilhada e participativa. Desenvolvimento $e$ Meio ambiente, 11.

Kumar, L. and Mutanga, O. (2019). Google Earth Engine Applications. MDPI.

Mota, F. P., Mateus, M. G., and Aguiar, Marilton S.and Adamatti, D. F. (2020). Google earth engine e sua aplicabilidade na gestão de recursos hídricos. Remote Sensing, 1.

Mutanga, O. and Kumar, L. (2019). Google earth engine applications.

Nguyen, U. N., Pham, L. T., and Dang, T. D. (2019). An automatic water detection approach using landsat 8 oli and google earth engine cloud computing to map lakes and reservoirs in new zealand. Environmental monitoring and assessment, 191(4):235.

Shami, S. and Ghorbani, Z. (2019). Investigating water storage changes in iran using grace and chirps data in the google earth engine system. International Archives of the Photogrammetry, Remote Sensing \& Spatial Information Sciences.

Wang, C., Jia, M., Chen, N., and Wang, W. (2018). Long-term surface water dynamics analysis based on landsat imagery and the google earth engine platform: A case study in the middle yangtze river basin. Remote Sensing, 10(10):1635.

Xia, H., Zhao, J., Qin, Y., Yang, J., Cui, Y., Song, H., Ma, L., Jin, N., and Meng, Q. (2019). Changes in water surface area during 1989-2017 in the huai river basin using landsat data and google earth engine. Remote Sensing, 11(15):1824. 